\title{
New approach to modeling the nonlinear loads
}

\author{
L.I. Kovernikova ${ }^{1,2, *}$, Van Chung Luong ${ }^{2}$ \\ ${ }^{1}$ Melentiev Energy Systems Institute SB RAS, Irkutsk, Russian Federation \\ ${ }^{2}$ Irkutsk National Research Technical University, Irkutsk, Vietnam
}

\begin{abstract}
Parameters of operating conditions of electric power systems shall ensure efficient and reliable operation of both power generating companies and of consumers, which to a great extent depends on power quality. Most urgent problem in this area is currently non-sinusoidal voltage. Power quality control, development of technical measures to sustain parameters of harmonic modes in conformity with provisions of GOST 32144-2013 require calculations that, in turn, need models adequately representing the electric networks and nonlinear loads in the computing program. Results of studies on parameters of harmonic modes that were obtained by measurements have shown that harmonic modes are random, and their parameters have probabilistic nature. Due to unpredictability of harmonic modes, nonlinear loads can be modeled using measured parameters. The paper presents methodological approach, algorithms and a computing program for modeling the nonlinear loads using parameters measured in the nodes of their connection to the power supply, and gives examples of modeling the nonlinear loads.
\end{abstract}

\section{Introduction}

Parameters of electric power systems conditions shall ensure efficient and reliable operation of both power generating companies that supply power to consumers, and of consumers, which to a great extent depends on power quality. Low power quality raises power losses in the electric networks, reduces service life of electric equipment, and efficiency of process equipment of industrial enterprises. Non-sinusoidal voltage is currently a challenge related to power quality [1]. This problem is urgent not only for Russia, but for other countries as well. The amount of nonlinear loads (sources of current harmonics) is rapidly and continuously growing. Levels of current and voltage harmonics in the electric networks grow as well.

For controlling the non-sinusoidal modes in the electric networks, for their analysis, for developing the technical measures to sustain the harmonic mode parameters in conformity with requirements [2], the harmonic modes need to be calculated. Calculations can be performed provided the models adequately representing electric networks and nonlinear loads are used for modeling the harmonic modes. Nonlinear load models have been developed for a long time. Both Russian and foreign scientists have made considerable contribution into the theory of modeling the nonlinear loads [3-5].

Results of studies on parameters of harmonic modes have shown that harmonic modes are random, and their parameters have probabilistic nature [6-8]. They depend on the network configuration, structure of its elements, on the amount of nonlinear loads, wave and frequency properties of the network, on values of phase angles of harmonic currents of nonlinear loads, on voltage values on the clamps of nonlinear equipment, on changes of operating modes and loads, and many other factors. Due to their unpredictability, the nonlinear loads can be modeled based on measurements of harmonics only.

Calculation of non-sinusoidal mode of an electric network is an aggregation of mode calculations for harmonics from 2 to 40 in conformity with [2]. In practice, the number of harmonics can be lower, which is determined in the result of analysis of measured values of current and voltage harmonics. Calculations at each harmonic are performed using a system of equations for nodal voltages

$$
U_{n}=Z_{n} I_{n}
$$

where $n$ - harmonic number, $\boldsymbol{U}_{\boldsymbol{n}}$ - a column-matrix of values of nodal voltages to be determined, $\boldsymbol{Z}_{\boldsymbol{n}}-$ a square matrix of self- and mutual impedances of the network nodes, $\boldsymbol{I}_{\boldsymbol{n}}$ - a column-matrix of currents in the nodes that must be determined based on the results of measurements.

Each element of matrix $\boldsymbol{I}_{\boldsymbol{n}}$ is a complex number $I_{n i}=I_{a n i}+j I_{r n i}$, where $i-$ row number of the matrix corresponding to the node number of the network; $I_{a n}-$ active current of $n$-th harmonic; $I_{r n}$ - reactive current of $n$-th harmonic. Active and reactive currents are computed based on the measured parameters as

$$
\begin{aligned}
& I_{a n}=I_{n} \cos \varphi_{U I(n)} \\
& I_{r n}=I_{n} \sin \varphi_{U I(n)}
\end{aligned}
$$

\footnotetext{
"Corresponding author: kovernikova@isem.irk.ru
} 
where $I_{n}-$ measured rms value of the $n$-th harmonic current; $\varphi_{U I(n)}$ - measured value of the phase angle between voltage and current of the $n$-th harmonic. Currents $I_{a n}$ and $I_{r n}$ similarly to measured ones $I_{n}$ and $\varphi_{U I(n)}$, are series of random values $X: x_{1}, x_{2}, \ldots, x_{i}, \ldots$, $x_{m}$, where $m$ is the number of elements in the series. A model of nonlinear load is a set of distribution functions for currents $I_{a n}$ and $I_{r n}$ that allow computation of values of active and reactive current harmonics of nonlinear loads with desired probability. For solving the system (1) in conformity with [2], probability of currents values shall be $95 \%$.

Subject of the present paper is methodological approach to modeling the nonlinear loads that takes into account features of parameters of harmonic modes in the electric networks, an algorithm for modeling the distribution functions of active and reactive current harmonics, an algorithm of a computer program for identification of distribution functions of random values of current harmonics and computation of their values with the desired probability, it also gives examples of using the developed methodological approach and computer program.

\section{Methodological approach to modeling the nonlinear loads}

Methodological approach takes into account features of parameters of harmonic modes [6-9] and includes: 1) check of measured series of random values of harmonic mode parameters for availability of abnormal elements (rough outliers), whose values considerably exceed the values of the remaining elements; 2) analysis of measured parameters of harmonic modes for determination of time intervals characterized by different values of parameter; 3 ) analysis of correlation relations between current and voltage harmonics; 4) analysis of coefficients of harmonic components of voltages ( $\left.K_{U(\mathrm{n})}\right)$ for selecting the harmonics for modeling; 5) analysis of directions of harmonic flows of active and reactive currents through a node connecting to the network of nonlinear load; 6) modeling the distribution functions of harmonics of active and reactive currents; 7) check of correctness of computed values of active and reactive currents with desired probability. The methodological approach is described further.

1) The series of measured parameters of the harmonic mode may contain elements whose values are many times different from the values of other elements (rough outliers). They distort the picture of the mode, do not allow constructing a real histogram and correctly determine the law of the distribution function. Outliers can be visually determined using scatter plot. When processing the results of measurements they are replaced with neighboring elements, a mean value of neighboring elements or by other ways proposed in [10-11].
2) Analysis of measured data demonstrated that the measured values of harmonic mode parameters notably differ from one another on some time intervals. Those differences are caused by changes in the network structure, in the parameters of its elements, in loads, etc., that occur during operation. In case of considerable differences in parameters on different time intervals the period of measurements shall be divided into intervals for which models of nonlinear loads should be developed.

3) Identification of availability or lack of correlation relationships between current and voltage harmonics allows use of appropriate mathematical methods during modeling. Lack of strong correlation relationship proves independence or weak dependence of values of voltage harmonics on current harmonics, i.e., evidences probabilistic nature of both parameters. Analysis of measured parameters of harmonic modes [7-8] confirmed that there is either no correlation between current and voltage harmonics or it is negligible. Lack of correlation or weak dependence is accounted for by the fact that measured parameters of harmonic modes are averaged values on some time interval. In [2] the averaging interval is 10 minutes.

4) Not all the harmonics out of the interval from 2 to 40 can be modeled, but only harmonics the value $K_{U(\mathrm{n})}$ of which exceeds limit values [2]. When selecting the harmonics for modeling, the measured values $K_{U(\mathrm{n})}$ should be compared to limit values for $95 \%$ and $100 \%$ of measurement time.

5) The values of current harmonics with numbers $\mathrm{n}>1$ are much smaller than the values of current harmonics for $n=1$. Direction of their flow may change due to a lot of insignificant causes for the first harmonic. Therefore, for each time interval defined in Item 2) the directions of flow of active and reactive current harmonics through a node for connection of a nonlinear load to electric network shall be analyzed. Analysis can be performed using a phase angle between harmonics of voltage and current $\varphi_{U I(\mathrm{n})}$ [12]. It is assumed that the active current has a positive sign if it flows from the network to the load. For the reactive current, the same direction is taken as for the reactive power of the load, which is inductive. Active and reactive currents flow from the network to the load if angle $\varphi_{U I(n)}$ lies in the first quadrant of the complex plane. Active current flows from the load to the network, whereas reactive current flows from the network to the load if angle $\varphi_{U I(n)}$ lies in the second quadrant. Both currents flow from the load to the network if angle $\varphi_{U I(n)}$ lies in the third quadrant. Active current flows from the network to the load, whereas reactive current flows in the reverse direction if angle $\varphi_{U I(n)}$ lies in the fourth quadrant. Thus, intervals of modeling identified in Item 2) can be further divided into four time intervals (variants) corresponding to positions of angle $\varphi_{U I(n)}$ on the complex plane. Variants with prevailing number of measurements (out 
of their total number of measurements) are preferable for modeling.

6) Identification of distribution functions of active and reactive current harmonics for computation with $95 \%$ probability or any other. Analysis of numerous measurements of current harmonics allows us to conclude that two different approaches (represented as two algorithms in Fig. 1) must be used for identification of distribution functions.

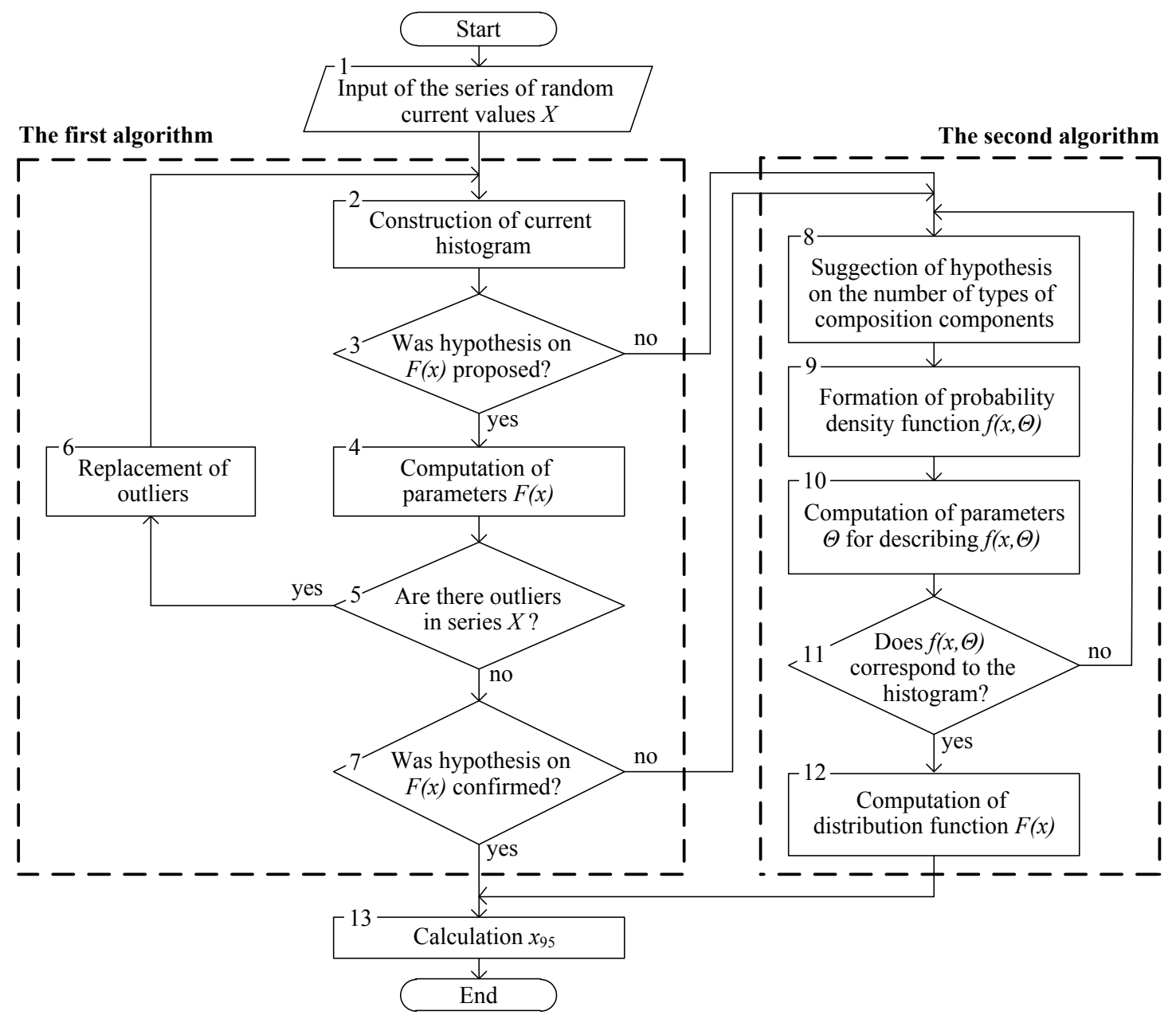

Fig. 1. Algorithms for modeling a current harmonics.

Notation: $X-$ a series of measured random values of current harmonic, $F(x)$ - a distribution function, $f(x, \Theta)$ - probability density function, $x_{95}$ - value of current harmonic with $95 \%$ probability.

The first algorithm (Blocks 2-7) is intended for identification of distribution functions of series $X$ corresponding to well-known distribution laws. The second algorithm (Blocks 8-12) is intended for identification of distribution functions of series $X$ corresponding to compositions of known distributions. Computer program has been developed on the base of algorithms presented.
7) After determination of $I_{a n}$ and $I_{r n}$ the correctness of values obtained shall be checked. To this end, the current value $I_{n}$, is computed using $I_{a n}$ and $I_{r n}$, and it shall not go beyond the measured currents.

\section{An algorithm of computer program for modeling the current harmonics}

A diagram of the computational algorithm is given in Fig. 2. It was developed on the base of algorithms for modeling the current harmonics given in Fig. 1. 


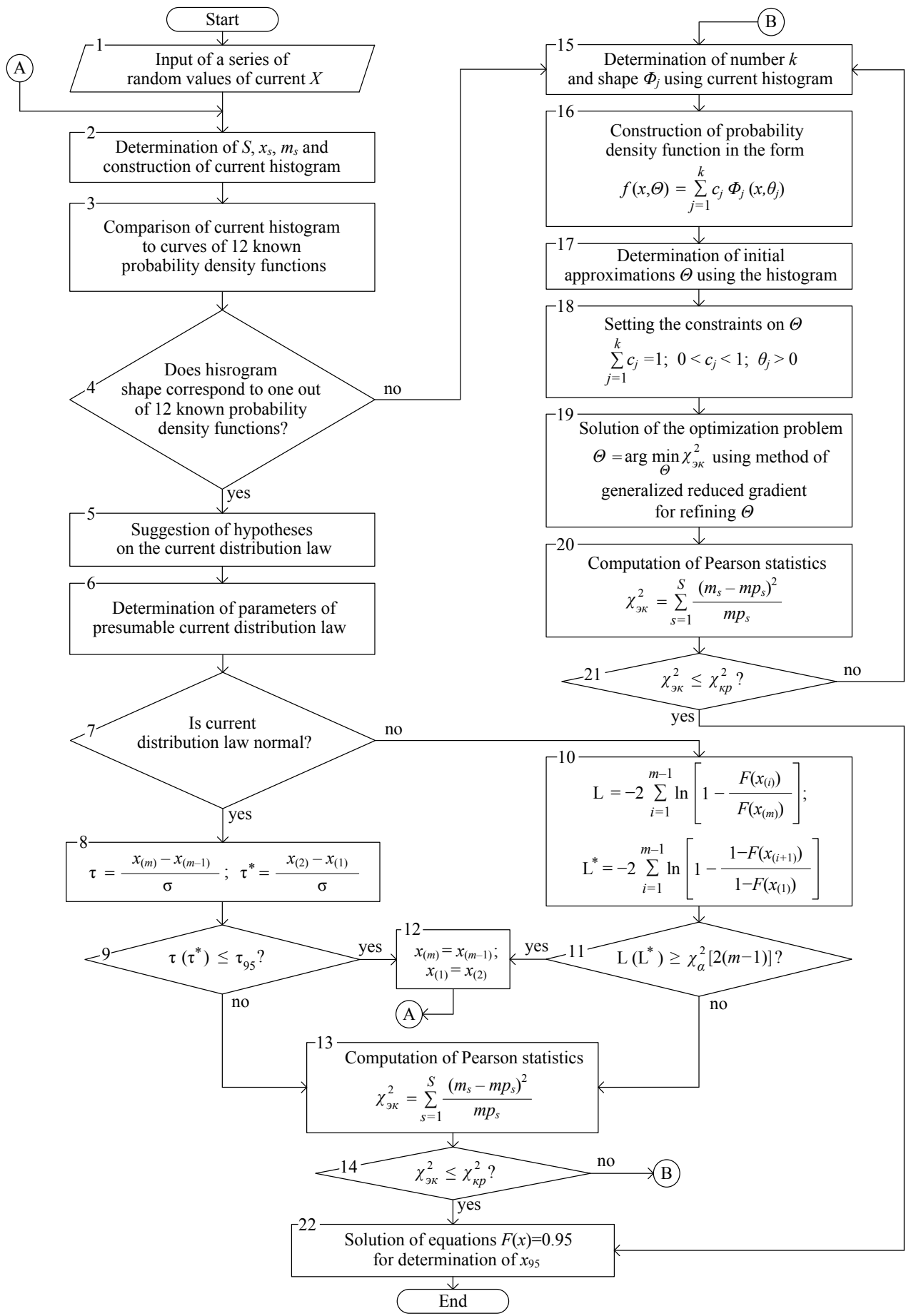

Fig. 2. Block-diagram of computer program.

Computer program contains the following blocks.

Block 1. Input to the program of a series of active and reactive current harmonics $X: x_{1}, x_{2}, \ldots, x_{i}, \ldots, x_{m}$, where $m$ - the number of elements in the series.

Block 2. Determination of the number of intervals of the histogram $(S)$; their boundary values $\left(x_{S}\right)$; the number of values $X$ in the series that occurred in the $s$-th interval $\left(m_{s}\right)$; histogram construction.

Blocks 3-5.Visual comparison of the shape of the current histogram to the shape of curves of twelve probability density functions used in the computer program to hypothesize the law of current distribution.
Twelve laws are proposed to be used in the procedure: Gaussian law, Weibull law, exponential law, gammadistribution, Rayleigh law, logarithmically normal law, beta-distribution, minimum value distribution law, maximum value distribution law, logistic law, and Maxwell and Cauchy distribution law. If the shape of the histogram does not allow hypothesizing the distribution law then go to Block 15. If current distribution law has been hypothesized, the hypotheses shall be checked following the algorithm by going over to Block 6 .

Block 6. Computation of parameters describing the proposed distribution law of the series $X$. For example, Gaussian distribution is described by two parameters: 
mathematical expectation $(\mu)$, and standard deviation $(\sigma)$.

Block 7. Selection of a criterion for checking the series $X$ for availability of outliers depending on the presumable distribution law.

Methodological approach already contains Item 1) for detection and replacement of rough outliers. But in the series $X$ can be elements that are not included into a compact group of the main mass of series elements and, at the same time, they are slightly removed from it. Such elements can be referred to as presumable outliers. They cannot be seen on the scatter plot. For detecting such outliers special statistical criteria shall be applied [10]. If presumable distribution law of the current is normal, then the availability of outliers can be checked using Irwin criterion, Von Neumann test, and others. If presumable law differs from normal one, then modification of Darling criterion is used [10].

Blocks 8-11. Check if there are the large $x_{m}$ and very small $x_{1}$ values of the series $X$ ordered in ascending order using the Irwin criterion (for that purpose statistics $\tau, \tau^{*}$ are computed and compared to critical value $\tau_{95}$ ) or using the Darling criterion modification (for that purpose statistics $\mathrm{L}, \mathrm{L}^{*}$ are computed and compared to the value of the $\alpha$-quantile of $\chi^{2}$-distribution.

Block 12. Replacement of outliers by neighboring elements of the series $X$ and then to Block 2, as replacement of outliers may change the shape of a histogram.

Blocks 13-14. Check of concordance of presumable current distribution with the histogram using Pearson goodness-of-fit criterion.

Blocks 15-21. Identification of probability density function of the series $X$ using method of separating mixtures of probability distributions [13] that is used when distribution of series $X$ cannot be described by known functions due to its complexity. The amount of components in the distribution composition and types of their probability density functions used in the computational procedure are hypothesized based on the histogram shape. Then the probability density function is formed as a weight sum of components of distribution composition

$$
f(x, \Theta)=\sum_{j=1}^{k} c_{j} \Phi_{j}\left(x, \theta_{j}\right),
$$

where $f(x, \Theta)$ - a desired function of the current probability density; $k \geq 1-$ a positive integer; $\Phi_{j}-$ a known function of probability density of the $j$-th component of the composition; $\Theta=\left(c_{1}, \ldots, c_{k}, \theta_{1}, \ldots, \theta_{k}\right)$ - a vector of parameters of composition components that are to be determined; $c_{j}$ - weighting factor of the $j$-th component, $c_{j}>0, j=1, \ldots, k, c_{1}+\ldots+c_{k}=1 ; \theta_{j}-$ a vector of parameters of the $j$-th component of the composition.

The task of distribution description consists in determination of the vector $\Theta$. Initial approximations of parameters of the vector $\Theta$, determined in the course of visual analysis of the histogram are defined using the series $X$.

A histogram of current with two peaks is given in Fig. 3 as an example. A hypothesis is suggested that a histogram has two components whose probability density functions follow Gaussian distribution.

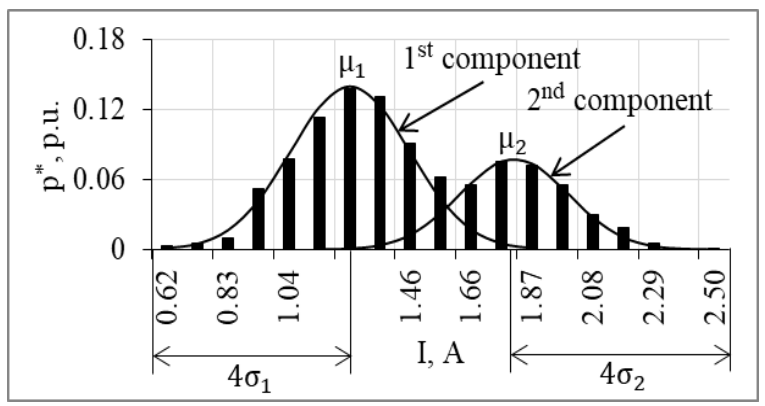

Fig. 3. A histogram of current with two peaks.

Vector $\theta_{j}$ consists of two parameters: $\theta_{1}=\left(\mu_{1}, \sigma_{1}\right)$, $\theta_{2}=\left(\mu_{2}, \sigma_{2}\right)$, and vector $\Theta$ consists of six parameters, i.e., $\Theta=\left(c_{1}, c_{2}, \mu_{1}, \mu_{2}, \sigma_{1}, \sigma_{2}\right)$. Initial values $\mu_{1}, \sigma_{1}$, $\mu_{2}, \sigma_{2}$ are determined from the histogram. For determination of $c_{1}, c_{2}$ the total area of the histogram under the curves of composition components is assumed to be equal to 1 . Values $c_{1}, c_{2}$ are set equal to fractions of the areas under the curves of components.

At normal distribution the position of histogram peaks relative to the abscissa axis allows determination of mathematical expectation for each component. A half of the figure width under the curve of the component allows determination of standard deviation [14].

Further refinement of $\Theta$ is performed by solving the optimization problem

$$
\Theta=\arg \min _{\Theta} \sum_{s=1}^{S} \frac{\left(m_{s}-m p_{s}\right)^{2}}{m p_{s}}
$$

subject to constraints

$$
\begin{gathered}
g_{1}(\Theta)=\sum_{j=1}^{k} c_{j}-1=0, \\
h_{1}(\Theta)=-c_{j}<0, \\
h_{2}(\Theta)=c_{j}-1<0, \\
h_{3}(\Theta)=-\theta_{j}<0,
\end{gathered}
$$

where $p_{s}$ - theoretical probability of occurrence of random values of series $X$ into $s$-th interval; $m p_{s}-$ theoretical frequency of occurrence of random values of series $X$ into the $s$-th interval.

Block 22. Computation with the given probability, e.g., $95 \%$, of value of current harmonic by solving the equation $F(x)=0.95$. The modeling of one harmonic of active or reactive current of nonlinear load terminates with computation of value of the current harmonic. Computer program has been implemented in MS Excel 
and Visual Basic programming environment for Windows-applications [15].

\section{An example of modeling the current harmonics at the nodes of traction transformers connection to the network}

Railway substations with traction transformers get power from $110-220 \mathrm{kV}$ public distribution network. Each substation has two 40 MWA transformers. One transformer is backup. $25 \mathrm{kV}$ winding of a transformer powers the electric-traction network that powers electric locomotives. Locomotives are operated by DC engines powered from single-phase two-pulse rectification circuit that originate current harmonics penetrating into the power network through transformers.

Modeling the 3-rd and 5-th harmonics of active and reactive currents at the nodes of traction transformers connection to the network at four substations of EastSiberia Railway (Mysovaya, Tataurovo, Zaigraevo, Novo-Il'insk) has shown that laws of harmonics distribution of active and reactive currents obey the known distribution laws: Gaussian law, exponential law, gamma-distribution, Weibull law, and have more complicated forms that are compositions of known distribution laws.

\section{Conclusions}

Methodological approach, algorithms of modeling, computer program algorithm, and computer program for modeling nonlinear loads using the measured parameters of harmonic modes in nodes connecting nonlinear loads to the electric network, which allow representing nonlinear loads as a set of distribution functions of current harmonics, has been developed.

Third and fifth current harmonics at the nodes of traction transformers connection to the network at substations of East-Siberia Railway were studied and modeled. The results have shown that the harmonics of the currents are distributed according to various laws: Gaussian law, exponential law, gamma distribution, Weibull law, and are also described by compositions of known distribution laws.

The research is conducted in the framework of the research projects № AAAA-A17-117030310432-9 and № AAAA-A17$117030310438-1$ of the program of fundamental research of SB RAS III.17.4.

\section{References}

1. L.I. Kovernikova, V.V. Sudnova, R.G. Shamonov, et al., Electric power quality: present-day state, problems and proposals to their solutions (Nauka, 2017) (in Russian)

2. State standard 32144-2013. Electric energy. Electromagnetic compatibility of technical equipment. Power quality limits in public power supply systems (2014) (in Russian)
3. I.V. Zhezhelenko, High harmonics in the power supply systems of industrial plants (Energoatomizdat, 2000) (in Russian)

4. J.D. Greene, C.A. Gross, Nonlinear Modeling of transformers, IEEE Transactions on Industry Applications, Vol. 24, No. 3, 434-438 (1988)

5. Probabilistic aspects task force of the harmonics working group subcommittee of the transmission and distribution committee, "Time-varying harmonics: Part I, characterizing measured data”, IEEE Transactions on Power Delivery, Vol. 13, No. 3, 938-944 (1998)

6. E.W. Kazibwe, T.H. Ortmeyer, M.S.A.A. Hammam, Summation of probabilistic harmonic vectors, IEEE Transactions on Power Delivery, Vol. 4, No. 1, 621-628 (1989)

7. L.I. Kovernikova, Some results of research into harmonics in the high voltage networks with distributed nonlinear loads, Przeglad Elektrotexchniczny 11, 239-243 (2013)

8. L.I. Kovernikova, Some properties of harmonics mode parameters in electrical network with distributed nonlinear loads, Proceeding of International Conference "Power Quality Management", 101-108 (Moscow, 2014) (in Russian)

9. L.I. Kovernikova, Van Chung Luong, Algorithm for high-voltage network nonlinear loads modeling on measured parameters, Electrichestvo 10, 16-25 (2017) (in Russian)

10. A.I. Kobzar, Applied mathematical statistics. For engineers and researchers (M.: FIZMATLIT, 2012) (in Russian)

11. B.Yu. Lemeshko, S.B. Lemeshko, S.N. Postovalov, E.V. Chimitova, Statistical data analysis, simulation and study of probability regularities. Computer approach (Novosibirsk: NSTU Publisher, 2011) (in Russian)

12. A.E. Emanuel, Power definitions and physical mechanism of power flow (John Wiley \& Sons, 2010)

13. O.K. Isaenko, V.Yu. Urbakh, Division of probability distribution mixtures into their components, Itogi nauki i techn. Ser. Probability Theory. Math. Stat. Theor Cybern., V. 13, 37-58 (VINITI, M., 1976) (in Russian)

14. L. Zacks, Statistical estimation (Moscow, "Statistics", 1976) (in Russian)

15. State Registration Certificate of Computer Program No. 2019619614. Software for modeling the current harmonics of nonlinear loads using measured parameters. (in Russian) 